\title{
Thermal Simulation of Power-Controlled Micro-CHP Systems for Residential Buildings
}

\author{
Sebastian Stinner Dirk Müller \\ Institute for Energy Efficient Buildings and Indoor Climate \\ E.ON Energy Research Center, RWTH Aachen University \\ Mathieustr.10, 52074 Aachen, Germany \\ sstinner@eonerc.rwth-aachen.de
}

\begin{abstract}
Combined heat and power (CHP) plants are a wellknown technology for industrial and district heating appliances. As those plants are often used to optimally satisfy thermal demands they often run heatcontrolled. The power generation profiles of those plants are badly predictable. Those badly predictable power generation profiles are fluctuating and central power plants have to work in the times when the heatcontrolled plants do not run. Due to these circumstances it should be analysed to what extent a powercontrolled operation can be applied. For this purpose a dynamic simulation of the whole system is necessary. This paper presents the possibilities of a dynamic simulation of a one-family-house with a power-controlled micro-CHP unit and a thermal storage.
\end{abstract}

Keywords: CHP; electrical grid; grid compatibility

\section{Introduction}

The energy supply of Germany will change significantly within the next few years. An increasing part of the power supply will be based on fluctuating sources like wind power or photovoltaics. On the other hand, about 35 per cent of the final energy in Germany is used for space heating and domestic hot water in buildings [3]. Thus, reasonable concepts for the building sector have to be found.

In periods where there is not enough power supply from the regenerative sources, flexible and energy efficient alternatives have to be considered. One of these alternatives could be combined heat and power (CHP) plants. The waste heat of the power generation process is used at the same time for example for space heating and domestic hot water. In many cases these plants can work more efficient than other options to generate heat. To combine the advantages of the CHP-plant as a fast reacting power generator and as an efficient heat supply system, those plants should be operated in a power-controlled way. This includes that a thermal storage is needed to buffer the discrepancy between the run times of the CHP plants and the thermal demand in the building.

Because of the increasing dynamics of those systems, dynamic simulations have to be considered to evaluate which role a system with power-controlled CHP-plants can play in the future. For these simulations, libraries are used that were developed at the Institute for Energy Efficient Buildings and Indoor Climate at RWTH Aachen University. They offer the possibility to simulate the performance of the CHP plant and the storage system, the dynamic thermal characteristics of the building and the user behaviour [2] With this approach, an integrated evaluation of whole micro-CHP systems is possible.

\section{Definition of the problem}

Due to new regulations (e.g. [1]) and an increased awareness of energy topics in public, the integration of regenerative sources in the German power supply increases. Especially wind power and photovoltaics are used as regenerative power sources. The disadvantage of these technologies is their non-controllability. They are completely dependent to weather conditions (wind speed and directions, solar radiation) and it is clear that there is a discrepancy between generation and demand.

At some times, there will be more regenerative energy generated than needed. At other times, the demand is higher than the generation from renewable energies. This would especially happen, if there are many consecutive days without wind and maybe nearly no direct solar radiation because of clouds and 
other effects. The arising gap between generation and demand should be closed with technologies that are as efficient as possible. (Micro-)CHP units, for instance, can reach higher efficiencies than pure power generating units (figure 1), and could be a suitable alternative to conventional power plants. Another aspect is

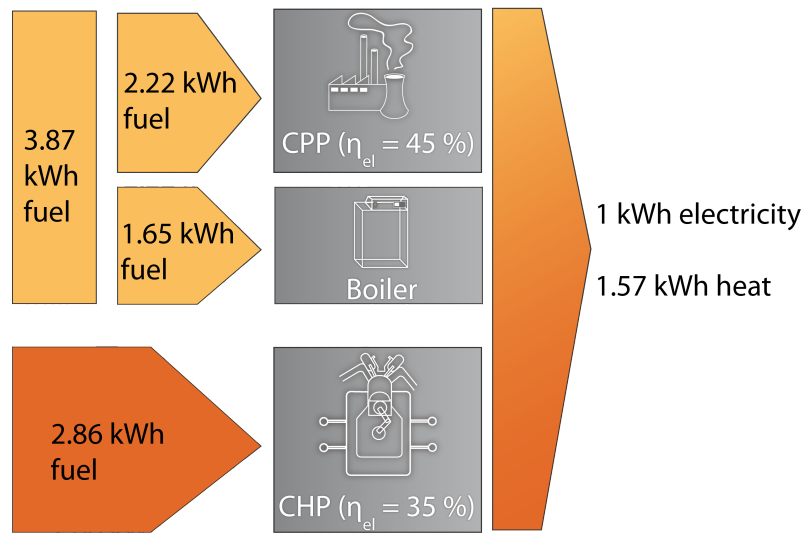

Figure 1: Comparison of combined heat and power generation and the separated generation [4]

the increasing dynamic of the system. Power plants are controlled with schedules that are generated from weather forecasts etc. The forecasts (one day before) and the real situation (e.g. solar radiation) can differ in a strong way (figure 2). This results in the need for fast reacting and fast starting and stopping technologies. For these applications, CHP units based on internal combustion engines are a suitable alternative because of their well controllable and fast reacting load conditions.

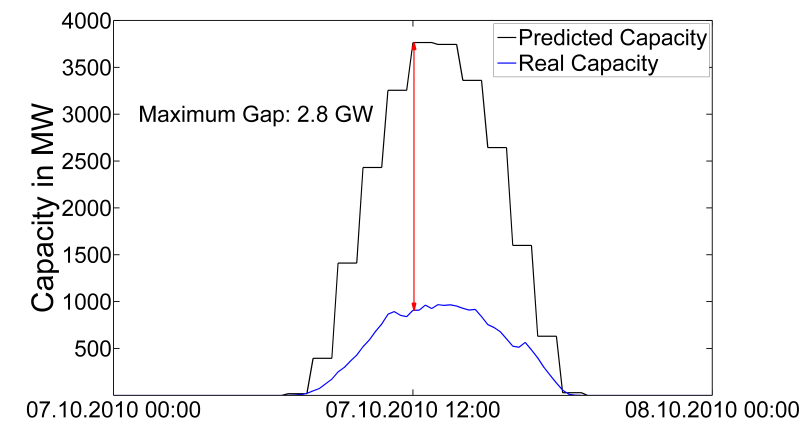

Figure 2: Comparison of predicted and real capacity of photovoltaics in the zone of one German grid operator [4]

For an efficient use of CHP units, the heat has to be used directly in the building. Residential buildings are predestinated as heat sinks for CHP plants, because of a year-round heat demand. This heat demand is signif- icantly larger in winter because of the heating demand on colder days. In summer there is just the demand for domestic hot water. Due to this situation and the fact that the thermal demand does not coincide with the generation (especially with a power-controlled device), thermal storages play an important role in such a concept. Thermal storages are cheap in comparison to electrical storages with a similar capacity. The size and insulation of those thermal storages has to be analysed to find out which kind of storage should be used.

Besides the storage, also the single CHP units have to be analysed. Today, there are several micro-CHP units with capacities of $1 k W_{e l}$ on the market for onefamily-dwellings. Based on internal combustion engines, they deliver a thermal power of about $2.5 \mathrm{~kW}$. If they are used in a power-controlled way, this could result in less delivered thermal power than would be needed for space-heating and domestic hot water supply. A solution to this problem could be an overdimensioning of the CHP plants, so that in times of a running plant, the thermal storage can be loaded very fast to provide a secure supply based on the CHP-plant as long as possible. For times, when a secure supply cannot be guaranteed with this stored thermal energy, a peak-load boiler should be installed.

Before those systems can be tested in reality in a power-controlled way, they should be analysed in simulations to get insight to most of the occurent effects. Because of the complexity and dynamics of this system, a coupled thermal, hydraulic and rudimentary electrical simulation is used. Modelica with its equation-based modelling approach is a good tool to bring this complexity into a model based on single components. The used model will be explained in the following chapter.

\section{Whole building system simulation}

\subsection{Design}

For the evaluation of power-controlled micro-CHPsystems, a whole system model is needed. For the hydraulic components, the Modelica fluid-library is used. With this library, easy connection setups between single components of the model are possible. Standard components like pipes, vessels and valves can be composed to new components. It is also possible to connect the different elements to a whole hydraulic circuit. The used medium in this model is obviously water. All the components are interacting with each other, so that it is very difficult to just simulate one compo- 
nent after the other. Especially the storage effects of the building mass and the included hot water storages can just be analysed in a coupled complete system simulation. The different models for simulation of powercontrolled and heat-controlled operation are shown in figure 3 .

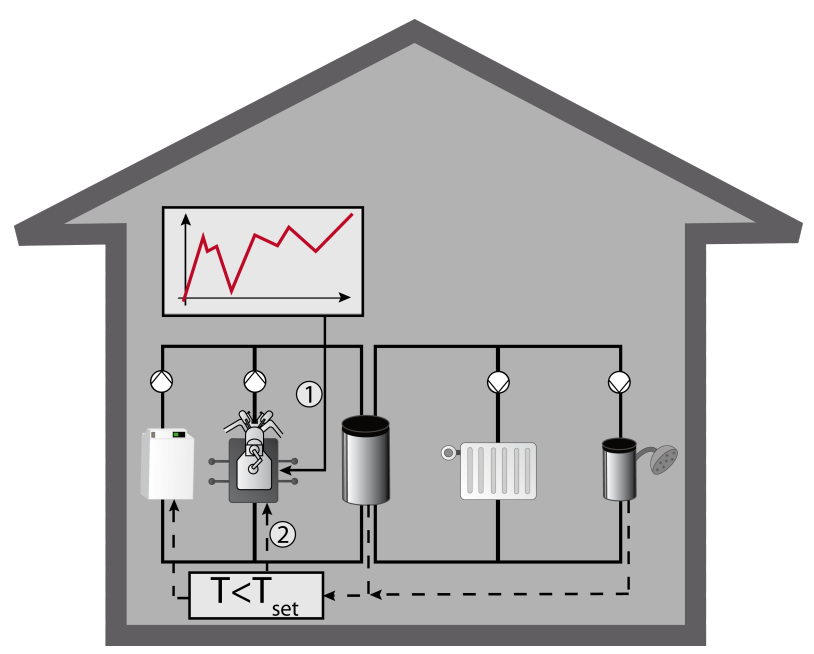

Figure 3: The whole building simulation model

The heat generating units are the CHP plant and the peak-load boiler. There are two storages included, one for space heating and one for domestic hot water. The boiler switches on if the stored heat cannot provide the heat supply in both heat-controlled and powercontrolled operation. The CHP plant is either running based on a predefined profile (power-controlled, control 1 in figure 3) or running due to a temperature drop in the storages (heat-controlled, control 2 in figure 3). The heat transfer to the single rooms is guaranteed with radiators.

The model of the micro-CHP plant is built up on manufacturer's data [7]. It is mainly based on predefined table values. This means that a given relation between electrical power, thermal power and fuel consumption can be set in the model for different load types (part load in different steps and full load). This simple approach of modelling a CHP-unit gives us the possibility to study the behaviour of the total building energy system.

Some delay elements are included to improve the dynamics of start-up and shut-down processes. Those processes are very important to model a powercontrolled CHP-unit. The model has an input to predefine the values forced by the electrical grid operator. This signal leads to a calculation of the belonging thermal power and fuel consumption. The calculated thermal power is fed to a volume element of the Modelica fluid library. If the plant runs heat controlled, it just switches off or modulates if a predefined outflow temperature is reached. In both cases (heat-controlled and power-controlled), a superior control can be implemented to switch the plant on or off. This can be used for security applications etc.

As stated before, a buffer storage has to be integrated to decouple the generated heat from the demand. This decoupling is necessary both in the heatcontrolled and in the power-controlled operation. The buffer storage is built up as a stratified storage with several layers which are thermally and hydraulically connected [6]. Besides the stored energy and the heat and mass transfer inside the storage, the heat losses to the environment (in the basement) have to be considered, because these losses will influence how long the stored energy can be used. The water elements inside the storage are volume elements of the Modelica fluid library. The fluid transfer between the layers is calculated automatically because of the connection to the hydraulic network of the building. The heat transfer between the layers is calculated with an approach of effective thermal conduction. For the heat transfer to the environment, a physical approach of heat transfer (convection, conduction, convection) in a tube is used. In the upper part of the domestic hot water storage, a heat exchanger is included to seperate the heat supply circuit physically from the domestic hot water which has to stay very clean.

Besides the supply system, also the building has to be modelled. In the examined system, the house is modelled physically to represent all the storage and loss effects that can be observed in a house. These are for example all transmission losses due to the temperature difference between inside and outside. Besides, the storage capacity of the walls is also considered. If a wall consists of different layers (e.g. concrete and insulation material), those different layers are implemented with their storage capacity and heat transport properties. Another important effect are the ventilation heat losses of the building effected by infiltration and air exchange through natural ventilation caused by the user of the building. The user behaviour is also important for the thermal simulation of the building, because a human being produces heat itself and uses different electrical devices which produce additional heat.

The third thing where user behaviour plays a role is the domestic hot water tapping profile. The impact of the domestic hot water supply on the overall heat supply for buildings will increase as the space heat demand will gradually decrease in refurbished and new buildings. The supply of the users with domestic hot 
water is implemented with a domestic hot water tank which is loaded by the CHP-unit and, if necessary, by the boiler. Another possibility to generate domestic hot water would be a fresh water station directly coupled to the buffer storage. This should be developed in the future.

To set the flow temperature of the space heating system to a desired value, a return addition is integrated. This element is especially interesting when there is demand for domestic hot water and space heating at the same time and the temperature level for the space heating is lower than the desired domestic hot water temperature. The building model includes heat valves that inhibit the fluid flow through the radiators if the desired indoor temperature is reached. The desired indoor temperature can be varied and is set from an input table. In the case described here, a temperature of $21^{\circ} \mathrm{C}$ during the day and $17^{\circ} \mathrm{C}$ during the night is set up. If the temperatures get higher than the desired value, the heat valves close and no fluid flows through the radiator anymore. As soon as the temperatures drop down, the valves open again and let the hot water pass. The power of the radiators is then calculated depending on the room temperature, the flow temperature, the surface area of the radiator, the nominal power and the radiator exponent.

\subsection{First results}

To show the possibilities of the model and the insights that can be obtained, two examples are shown. The first analysed plant has a maximum thermal power of about $2.5 \mathrm{~kW}$ th and a maximum electrical power of 1 $k W_{e l}$. This is a standard micro-CHP unit which can be bought on the German market. Two models will be compared. On the one hand this is a model with a heatcontrolled CHP-unit. This unit is only controlled by the temperatures in the buffer storage and the storage for domestic hot water. On the other hand, we analyse a power-controlled operation. In this second model, the times when the CHP unit runs are pre-determined by a certain profile that is set up maybe from a grid operator. Such a profile will result from a residual load profile. It is calculated for every time step as the difference between the electric load in the grid and the feed-in of renewable sources. If the residual load is above zero, some CHP units have to run because of a frequency drop in the grid. In times when the residual load is below zero, this energy has to be stored or maybe used in another way (in electricity-driven heat pumps for example). For two days, this profile is shown in figure 4 .

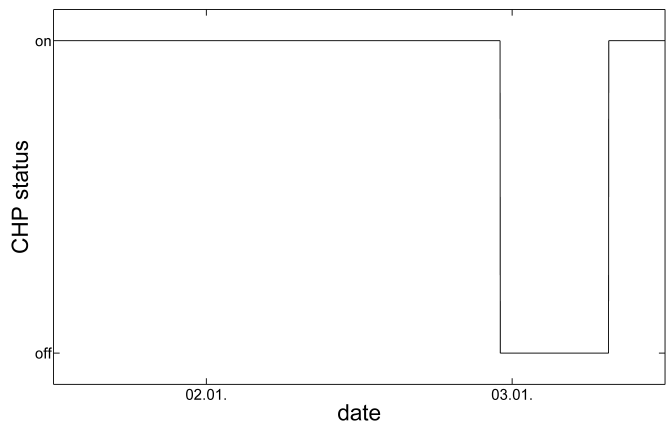

Figure 4: Desired on/off-profile of the CHP unit for two days

After running the simulation, the on/off-profile for the CHP-unit for two exemplary days is shown in figure 5 in the lower part. In contrast to that, the heatcontrolled CHP-plant runs nearly continuously for all the days with a little exception at the beginning of January 2nd. At the same time, the power-controlled CHP-unit switches off, although the electricity-profile in figure 4 is set to on at this time. Both switch-off processes are due to an increasing temperature in the storage which is shown in figure 5 in the upper part. The storage volume is set to 1000 litres for both cases.

During the night, there is nearly no heat consumption which leads to an increasing temperature in the storage and also an increasing temperature of the fluid flowing to the CHP unit. To analyse these types of feedback between different parts of the energy system, a dynamic approach as it is used with Modelica is needed. We cannot fill the stratified storage until its whole temperature is at the maximum reachable temperature, because the plant has also some limits. These limits have to be analysed in further activities. To guarantee a secure energy supply, the plants in the single houses have to interact with each other. In a case when one plant cannot operate anymore due to an increasing temperature, this can be detected at an earlier stage and another plant can run instead.

The second example which is presented is a system with a bigger engine with an electrical power of $3 \mathrm{~kW}_{e l}$ and a thermal power of $8 \mathrm{~kW}$ th . This plant is overdimensioned for the examined building, but we want to study how such a plant will behave in a heat supply system for one building. Due to the bigger thermal capacity of the plant, also a bigger thermal storage is included. This storage has a volume of 2500 litres. This storage volume is needed to guarantee longer run times of the plant. But, as we can see in figure 6, the storage capacity is not big enough to let the CHP-plant run 


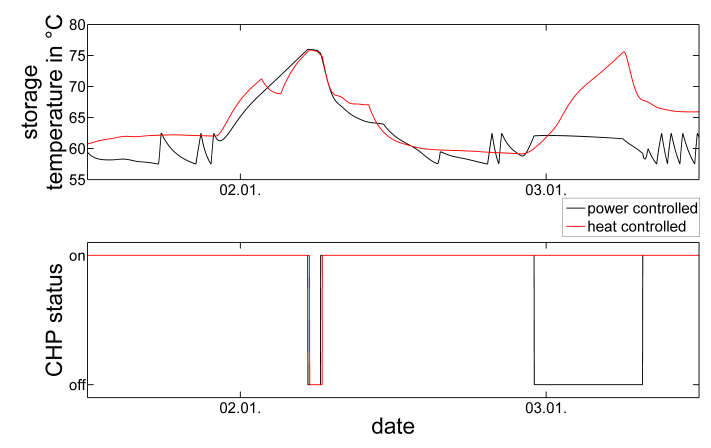

Figure 5: Temperature at the top of the buffer storage and the on/off-profile of the CHP unit

with the desired profile. The plant often runs in partload instead of full-load or it is even switched off. This shows that a system like this can just work in a house with worse insulation or it has to share the production of the desired electrical power with another house.

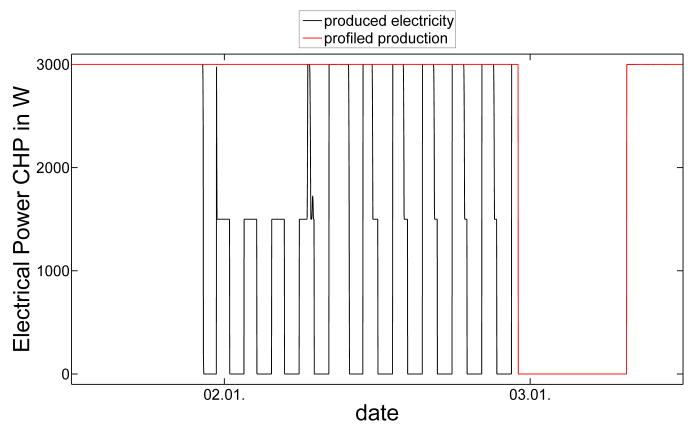

Figure 6: Desired profile and really produced electricity of the CHP unit ( $3 k W_{e l}$ ) for two days

Other effects like the behaviour of the peak-loadboiler can also be studied. In this example, the additional energy which should be provided by a peakload-boiler is analysed. For the heat-controlled plant, no additional heat from the boiler is needed in the considered time period of two days. For the powercontrolled plant with a capacity of $1 k W_{e l}$, an additional heat energy demand of about $44 \mathrm{kWh}$ is calculated. This shows, that this operation will lead to higher demands for boilers. The solution for this could be the over-dimensioned power-controlled plant with a capacity of $3 k W_{e l}$. With this plant, just a little demand of about $3 \mathrm{kWh}$ is calculated. With the approach of an over-dimensioned plant, we can decrease significantly the additional heat demand which is provided by a boiler. This does not regard the fact that the plant with $3 k W_{e l}$ was not running in the pre-defined way. With a plant running as it was pre-defined, the heat gained from the plant would increase significantly. If this operation would be shared to different houses as mentioned before, it would decrease again. Detailed analyses of these systems should follow.

In addition, the approach stated in this paper gives us the possibility to check, if the desired room temperatures are reached all the time with these new energy supply systems.

\section{Conclusion and outlook}

CHP plants can play a bigger role in the supply of residential buildings because of their flexibility and their energy efficiency. This paper shows, how Modelica can be used to model these new and distributed energy systems for the future. A model for the integration of the CHP plant in the heat supply for residential buildings is shown. The difference between heat-controlled and power-controlled operation modes is presented. Different user behaviour profiles can be included to improve the systems engineering adapted to the single user.

With this comprehensive model, a detailed analysis of future power-controlled micro-CHP-systems can be performed. Besides the analysis of power-controlled system, also switching between heat-controlled and power-controlled operation is possible and will be considered in the future.

Anyway, there are several things which should and will be implemented in the future. The model of the micro-CHP plants has to be validated with measurement data to be sure to represent the dynamics of the plant correctly.

The current models have to be simplified to less complex and less extensive models. This would provide the possibility to simulate more than one house in one model as it was mentioned in chapter 3.2. Thus, it would be possible to get the houses interconnected and to simulate the supply of whole city quarters with their electrical demand and fluctuating electricity sources like photovoltaics and wind power. This would give an integrated insight to the energetic impact of future energy systems with a higher rate of micro-CHPs.

A third point that has to be implemented is the interconnection of the different user profiles that are used in the model. In detail, these are the electrical demand profile, the domestic hot water profile, the natural ventilation profile and the heat source profile caused by internal loads. This would additionally improve the accuracy of the prediction for those energy systems. 


\section{References}

[1] EnEV 2009, Energieeinsparverordnung, Energy saving ordinance for buildings, 2009.

[2] A. Hoh, T. Haase, T. Tschirner, D. Müller. A combined thermo-hydraulic approach to simulation of active building components applying Modelica. In Proc. of 4th International Modelica Conference, Hamburg, March 2005.

[3] AG Energiebilanzen e.V., Anwendungsbilanzen für die Endenergiesektoren in Deutschland in den Jahren 2009 und 2010, 2011.

[4] TenneT TSO GmbH, Actual and forecast photovoltaic energy feed-in, http://www.tennettso.de/site/en/Transparency/ publications/network-figures/actual-andforecast-photovoltaic-energy-feed-in, last call: 11.05.2012.

[5] Aktualisierte und erweitere Testreferenzjahre (TRY) von Deutschland für mittlere und extreme Witterungsverhältnisse, Bundesinstitut für Bau-, Stadt- und Raumforschung, 2011

[6] K. Huchtemann, D. Müller, Advanced simulation methods for heat pump systems In Proc. of 7th International Modelica Conference, Como, Italy, September 2009

[7] P. Jahangiri, Simulation and Comparison of Different District Heating Networks in Combination with Co-generation Plants, Master thesis, 2010 